\title{
Tone sandhi of young Taiwanese speakers
}

\author{
Yuchau E. Hsiao*
}

\begin{abstract}
This paper addresses young Taiwanese speakers' tone sandhi. A young speaker's language is often a mixture of Taiwanese and Mandarin; though understanding Taiwanese, he/she frequently responds in Mandarin. This paper establishes a corpus of 'young Taiwanese', which reveals three different tone sandhi patterns from 'general Taiwanese'. First, a $\varphi$-break (sandhi domain break) may occur after a $X^{\text {head }}$. Second, a $\varphi$-break may occur after an adjunct XP. Finally, a $\varphi$ is restricted within three syllables. Young speakers seldom speak long Taiwanese expressions; they tend to break a long string into short fragments and match them with smaller syntactic or prosodic junctures.
\end{abstract}

Keywords. tone sandhi; prosody, XP; adjunct; Taiwanese; corpus

1. Introduction. This paper addresses the tone sandhi of young speakers of Taiwanese, a Southern Min dialect spoken in Taiwan, hereafter, Y-Taiwanese. The phonological behavior of the young speaker is worth exploring in two ways. First, a young speaker's language is often a mixture of Taiwanese and Mandarin in social environments. Second, a young speaker, though understanding Taiwanese, frequently responds in Mandarin, even at home. This paper builds a corpus of Y-Taiwanese, and engages in an analysis of the differences between Y-Taiwanese and the commonly known 'general Taiwanese' (as termed in Ang 2003), which refers to the accent spoken by the majority of population in Taiwan, hereafter, G-Taiwanese.

2. Some basics and previous studies. There are seven base tones in Taiwanese. The tone inventory includes 55, 33, 21, 53, 13, 3 and 5. In terms of Chao's (1930) tone transcription system, 5 represents high, 3 represents mid, and 1 represents low; 4 can be either high or mid and 2 can be either mid or low, depending on the tone inventory of a language. In (1), the tone of a smooth syllable is represented by two numbers: 55 stands for high level tone, 33 for mid level tone, 53 for high falling tone, 21 for low falling tone, and 13 for low rising tone. The single numbers, 3 and 5, indicate the tones of checked syllables. A smooth syllable is an open syllable or a syllable that ends in a sonorant. A tone carried by a smooth syllable is known as a smooth tone, or a long tone. A checked syllable ends in a glottal or unreleased voiceless stop, such as [?], [p], [t] and $[\mathrm{k}]$. A tone carried by a checked syllable is usually referred to as a checked tone, or a short tone. As the second mora of the checked syllable is occupied by a glottal or unreleased voiceless stop, the tone that docks on this mora is inaudible. All the tones surface with their corresponding sandhi forms in sandhi positions respectively. The tone inventory is listed in (1), and the relevant tone sandhi rule is given in (2).

(1) Tone inventory (Hsiao 2015: 144)

\begin{tabular}{|l|l|l|l|l|l|l|l|}
\hline Base tones & 55 & 33 & 21 & 53 & 13 & 3 & 5 \\
\hline Sandhi tones & 33 & 21 & 53 & 55 & 33 & 5 & 3 \\
\hline
\end{tabular}

\footnotetext{
* I thank the National Chengchi University Theoretical Phonology Laboratory team for their assistance, my four young informants for their recording, the participants at LSA 2018 for their comments and suggestions, and the Ministry of Science and Technology for sponsoring this research. Authors: Yuchau E. Hsiao, National Chengchi University (ychsiao@nccu.edu.tw).
} 
(2) Tone sandhi rule (Hsiao 1995: 68) ${ }^{1}$
$\mathrm{B} \longrightarrow \mathrm{S} /$
B (B: base tone; S: sandhi tone)

The rule in (2) indicates that given a pair of adjacent tones, the left tone occurs in its sandhi form, while the right tone retains its base form.

It has been observed in several works (Cheng 1968, Chen 1987, 2000, Hsiao 1991, Hsu 1994, among others) that the tone group (TG) that conditions tone sandhi in Southern Min dialects has direct or indirect access to syntactic maximal projections. Within a TG, only the rightmost tone retains its base form, while all the preceding tones are changed to their sandhi forms, regardless of the speech speed. ${ }^{2}$ Cheng (1968) suggested that Taiwanese tone sandhi is conditioned by NP, VP, S, etc., Drawing on evidence from Xiamen, Chen (1987) posits a cross-categorical generalization that the TG is marked at the right edge of a non-adjunct XP, but not of an adjunct XP, as in (3) and (4). ${ }^{3}$

(3) XP as a non-adjunct (Chen 1987: 122)

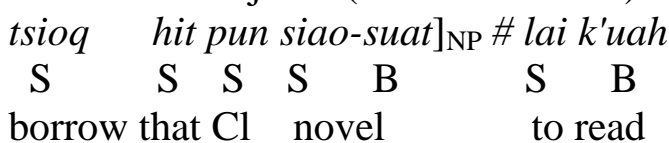

'borrow that novel to read'

(4) XP as an adjunct (Chen 1987: 124)

yi luan-tsu $]_{\text {AdvP }}$ kong

S $\quad$ S $\quad S \quad$ B

he mindlessly talk

'He is talking mindlessly'

The NP in (3) is an internal argument (object) of the verb tsioq 'borrow' but not an adjunct, and thus a TG marker (\#) exists after the NP, where only the rightmost syllable -suat preserves its base tone. On the other hand, The AdvP in (4) is an adjunct modifying the verb kong 'talk'; hence the adjunct XP does not block tone sandhi, but allows -tsu to carry a sandhi tone. However, Chen (1987: 144) hesitates to characterize the TG as a prosodic domain, but suggests that it "operates within a domain defined directly over surface syntax."

Selkirk (1986) proposes a set of end-based parameters which mark the break of a phonological phrase at the right or left edge of a syntactic XP or $X^{\text {head }}$. Hsiao $(1991,1995)$ observe that the TG is in fact not a syntactic constituent.

\footnotetext{
${ }^{1}$ The original rule is as follows: $\mathrm{T} \rightarrow \mathrm{T}$ ' $\mathrm{T}$, where $\mathrm{T}$ stands for base tone, and $\mathrm{T}$ ' for sandhi tone (cf. also Chen 1987; Hsiao 1991, among others).

${ }^{2}$ Shih (1986) indicates that Mandarin tone sandhi varies depending on the speed of speech; it refers to a smaller prosodic domain at a moderato or adagio tempo, such as foot, but refers to a bigger domain, such as intonational phrase, at a presto tempo. Taiwanese tone sandhi, however, is not affected by speech rate; the shape of TG remains the same at different tempos (Chen 1987; Hsiao 1991, 1995)..

${ }^{3}$ The adjuncthood holds between a head and its modifier. Chen's (1987) definition of adjunct is given below.

$\mathrm{XP}$ is an adjunct of $\mathrm{Y}$, if XP

a. appears in $[\ldots . \mathrm{XP} \ldots]_{\mathrm{YP}}$ and

b. is not a strictly subcategorized argument of $Y$

In his terms, an adjunct can be interpreted as a modifier, which is not an obligatory category but serves to modify its head
} 
(5) XP as a non-syntactic constituent (Hsiao 1991: 147)

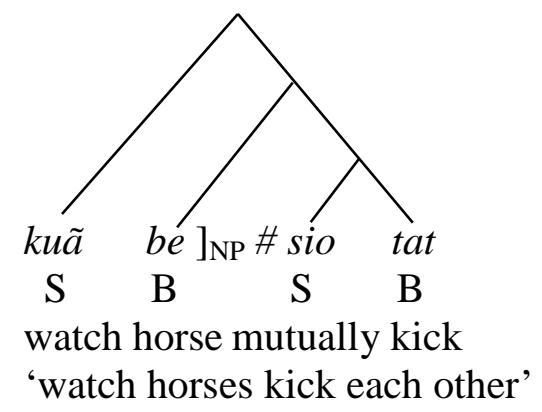

The phrase in (5) is parsed into two TGs, (kuã be) and (sio tat); however, the sequence (kuã be) does not form a syntactic constituent. Hsiao (1991) suggests that the TG is a prosodic domain, or more precisely, a phonological phrase, which in Selkirk's (1986) terms is marked at the right edge of a non-adjunct XP. Chen (2000) adopts this notion as well.

3. Data design. This research is concerned with the formation of tone sandhi domain at the syntax-phonology interface and the effect of prosody on tone sandhi. The data are thus collected through a careful design, based on the number of syllable and a variety of morphosyntactic structures. The data collection focusses on disyllabic, trisyllabic and tetrasyllabic constructions.

(6) Design of disyllabic constructions (S: sandhi target; T: trigger)

a. Designing the bracketed structures in different syntactic projections, such as NP, AP, VP, AdvP, etc. For example, $\left[[\mathrm{S}]_{\mathrm{AP}}[\mathrm{T}]_{\mathrm{N}}\right]_{\mathrm{NP}},\left[[\mathrm{S}]_{\mathrm{Advp}}[\mathrm{T}]_{\mathrm{A}}\right]_{\mathrm{AP}},\left[[\mathrm{S}]_{\mathrm{V}}[\mathrm{T}]_{\mathrm{NP}}\right]_{\mathrm{VP}},\left[[\mathrm{ST}]_{\mathrm{AdvP}}\right.$, and so Forth.

b. Designing different neighboring tones in the bracketed projections. For example, $\left[[\mathrm{S}]_{\mathrm{AP}}[\mathrm{T}]_{\mathrm{N}}\right]_{\mathrm{NP}}, \quad\left[[\mathrm{S}]_{\mathrm{AdvP}}[\mathrm{T}]_{\mathrm{A}}\right]_{\mathrm{AP}}, \quad\left[[\mathrm{S}]_{\mathrm{V}}[\mathrm{T}]_{\mathrm{NP}}\right]_{\mathrm{VP}}, \quad\left[[\mathrm{S}]_{\mathrm{V}}[\mathrm{T}]_{\mathrm{N}}\right]_{\mathrm{VP}}$

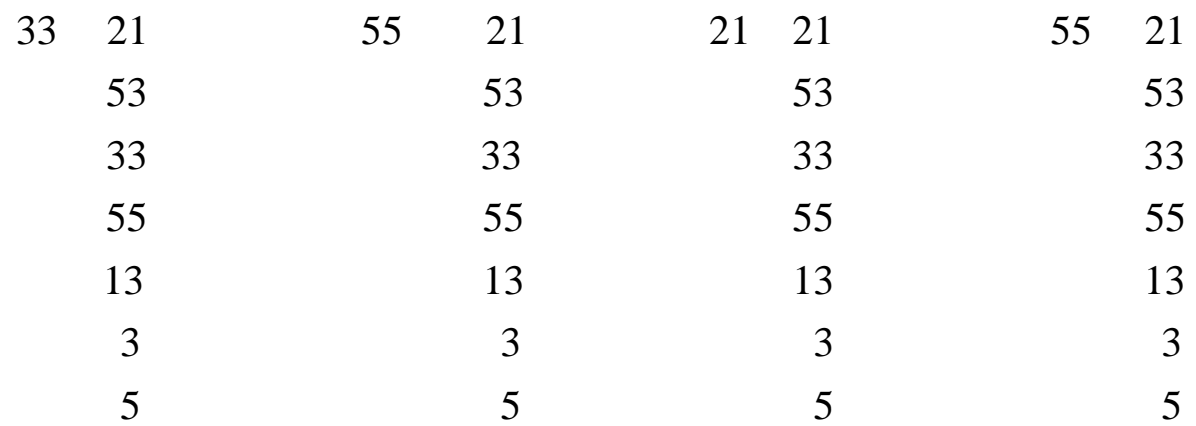

(7) Design of trisyllabic constructions ( $\mathrm{X}$ : any tone)

a. Designing data with different syntactic bracketings and placing the sandhi targets at different positions. For example, [[X.S] T], [S [T.X]], [S1[S2.T]], [[S1.S2]T], and so forth.

b. Designing the bracketed structures in different syntactic projections, such as S, NP, AP, and VP. For example, $\left[[\mathrm{X} . \mathrm{S}]_{\mathrm{AP}}[\mathrm{T}]_{\mathrm{N}}\right]_{\mathrm{NP}}$, [[S1 $\left.]_{\mathrm{NP}}[\mathrm{S} 2 . \mathrm{T}]_{\mathrm{V}}\right]_{\mathrm{VP}},\left[[\mathrm{X} . \mathrm{S}]_{\mathrm{AdvP}}[\mathrm{T}]_{\mathrm{A}}\right]_{\mathrm{AP}}$, $\left[[X . S]_{\mathrm{V}}[\mathrm{T}]_{\mathrm{NP}}\right]_{\mathrm{VP}},\left[[\mathrm{X} . \mathrm{S}]_{\mathrm{NP}}[\mathrm{T}]_{\mathrm{V}}\right]_{\mathrm{s}},\left[[\mathrm{S} 1]_{\mathrm{AdvP}}[\mathrm{S} 2 . \mathrm{T}]_{\mathrm{A}}\right]_{\mathrm{AP}},\left[[\mathrm{S} 1]_{\mathrm{AP}}[\mathrm{S} 2 . T]_{\mathrm{N}}\right]_{\mathrm{NP}}$, and so forth. 
c. Designing different neighboring tones in the bracketed projections. For example,

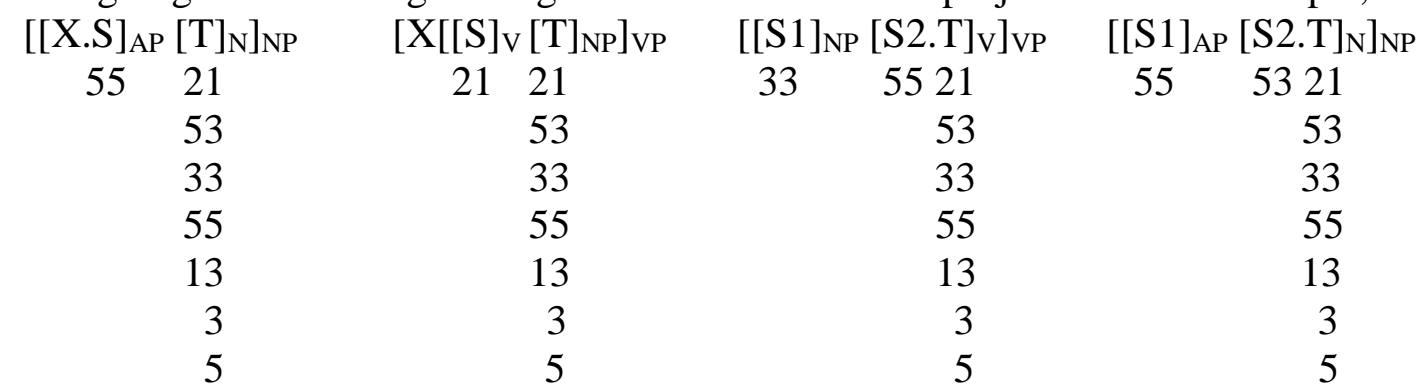

(8) Design of tetrasyllabic constructions

a. Designing data with different syntactic bracketing and placing the sandhi targets at different positions. For example, [[X.S][T.X]], [X[S[T.X]]], [[[X.X]S]T], [S1[S2[T.X]]], [S1[[S2.T]X]], [X[[S1.S2]T]], [[X.S1]S2]T], etc.

b. Designing the bracketed structures in different syntactic projections, such as S, NP, AP, and VP. For example, $\left[[X . S]_{\mathrm{AP}}[\mathrm{T} . \mathrm{X}]_{\mathrm{N}}\right]_{\mathrm{NP}},\left[[\mathrm{X} . S]_{\mathrm{AdvP}}[\mathrm{T} . \mathrm{X}]_{\mathrm{A}}\right]_{\mathrm{AP}},\left[[\mathrm{X} . \mathrm{S}]_{\mathrm{NP}}[\mathrm{T} . \mathrm{X}]_{\mathrm{V}}\right]_{\mathrm{S}}$, $\left.\left[\mathrm{X}\left[\mathrm{S}[\mathrm{T} . \mathrm{X}]_{\mathrm{NP}}\right]_{\mathrm{V}}\right]_{\mathrm{VP}},\left[\mathrm{X}\left[\mathrm{S} 2[\mathrm{~T} . \mathrm{X}]_{\mathrm{N}}\right]_{\mathrm{A}}\right]_{\mathrm{NP}},\left[\left[\mathrm{X}[\mathrm{S} 1 . \mathrm{S} 2]_{\mathrm{N}} \mathrm{T}\right]_{\mathrm{N}}\right]_{\mathrm{A}}\right]_{\mathrm{NP}}$, and so forth.

c. Designing different neighboring tones in the bracketed projections. For example, $\left[[X . S]_{\mathrm{AP}}[\text { T.X }]_{\mathrm{N}}\right]_{\mathrm{NP}}, \quad\left[[\mathrm{X} . \mathrm{S}]_{\mathrm{AP}}[\mathrm{T.X}]_{\mathrm{N}}\right]_{\mathrm{NP}}, \quad\left[[\mathrm{S} 1]_{\mathrm{NP}}[\mathrm{S} 2 . \mathrm{T} . \mathrm{X}]_{\mathrm{V}}\right]_{\mathrm{VP}}$,

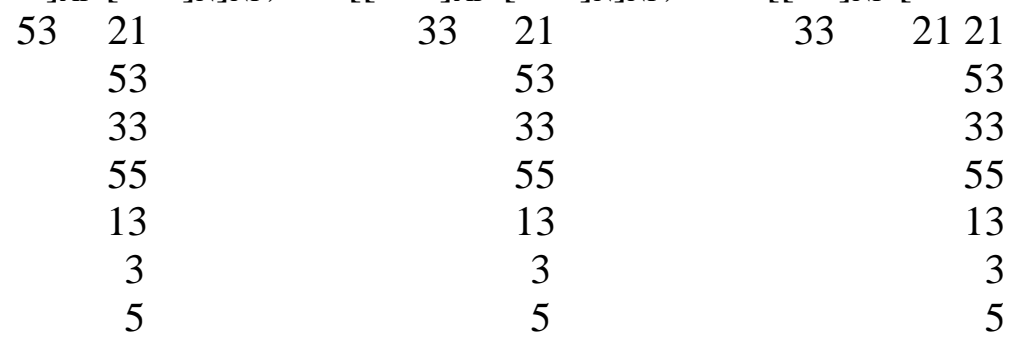

Disyllabic-to-tetrasyllabic expressions essentially include a basic variety of morphosyntactic structures that are crucial to any study of tone sandhi.

4. Corpus-based analysis. In this research, I establish a corpus of Y-Taiwanese, with the help of four young speakers, aged between 20 and 25. The corpus contains 923 words and phrases, from disyllabic to tetrasyllabic. According to the corpus, there are at least three tone sandhi patterns that are different from the commonly known G-Taiwanese. First, a phonological phrase break (hereafter, $\varphi$-break) may be found after a head verb. Consider (9a) and (9b).

(9). $\quad\left[\lim \left[[\mathrm{ng} \text { pui }]_{\mathrm{CLP}} t e\right]_{\mathrm{NP}}\right]_{\mathrm{VP}}$

drink two cup tea

'drink two cups of tea'

a. $(\lim n g$ pui te) $\varphi$

$S$ S S B

b. (lim) $\varphi($ ng pui te) $\varphi$

B S S B

In (9a), the entire VP constitutes a single $\varphi$, in which only te carries a base tone. This reading is the same as G-Taiwanese, found in only $56 \%$ of the VP's in the Y-Taiwanese corpus. In (9b) the verb lim 'drink' forms a $\varphi$ itself, and thus the VP is parsed into two $\varphi$ 's, (lim) and (ng pui te), where lim and te occur with their base tones. This reading is not acceptable in G-Taiwanese, but 
in the Y-Taiwanese corpus, $44 \%$ of the head verbs end in $\varphi$-breaks.

Second, a $\varphi$-break may also be found after an adjunct XP, as in (9c), where the verb and the classifier phrase ng pui 'two cups of' together form a trisyllabic $\varphi$.

c. (lim ng pui) $\varphi($ te $) \varphi$

$\mathrm{S} \quad \mathrm{S} \quad \mathrm{B} \quad \mathrm{B}$

In the corpus, $67 \%$ of the adjunct XPs end in $\varphi$-breaks.

Finally, the length of a $\varphi$ (or a tone sandhi domain) tends to be restricted within three syllables. Compare (10a) and (10b).

(10) a. (ng pui te) $\varphi$ 'two cups of tea'

$\mathrm{S}$ S B

b. (ng tua pui) $\varphi($ te $) \varphi$ 'two big cups of tea'

$S$ S B B

In the corpus, $52 \%$ of the trisyllabic constructions like (10a) form a single $\varphi$; on the other hand, $81 \%$ of the tetrasyllabic constructions like (10b) are parsed into two $\varphi s$. In general, young speakers do not speak long Taiwanese phrases or sentences often. When there is a chance to do so, they tend to break a long string into short fragments and match them with smaller syntactic or prosodic junctures.

5. Conclusion. This paper constructs a corpus of Y-Taiwanese, where the data are collected through a careful design, based on the number of syllable and a variety of morphosyntactic structures. The corpus shows some tone sandhi patterns different from G-Taiwanese. A $\varphi$-break may occur after a $X^{\text {head }}$ or an adjunct XP, and a $\varphi$ tends to be limited within three syllables. Young speakers seldom speak long Taiwanese expressions; they tend to break a long string into short fragments and match them with smaller syntactic or prosodic junctures. This research is a preliminary study of a two-year research project. Further investigation will be on restructuring of prosodic structures in relation to tone sandhi in Y-Taiwanese.

\section{References}

Ang, Wijin. 2003. The motivation and direction of sound change: on the competition of Minnan dialects Chang-chou and Chüan-chou and the emergence of general Taiwanese. Hsinchu: National Tsinghua University Dissertation.

Chao, Yuenren. 1930. A system of tone letters. Le Mâitre Phonétique 45. 24-47.

Chen, Matthew Y. 1987. The syntax of Xiamen tone sandhi. Phonology Yearbook 4. 109-150.

Chen, Matthew Y. 2000. Tone sandhi: patterns across Chinese dialects. Cambridge University Press, Cambridge.

Cheng, Robert L., 1968. Tone sandhi in Taiwanese. Linguistics 41, 19-42.

Hsiao, Yuchau E. 1991. Syntax, rhythm and tone: a triangular relationship. San Diego, CA: University of California Dissertation. Taipei: Crane Publishing Co., Ltd.

Hsiao, Yuchau E. 1995. Southern Min tone sandhi and theories of prosodic phonology. Taipei: Student Book Co., Ltd.

Hsiao, Yuchau E. 2015. Tonal chain shifts in Taiwanese: a comparative markedness approach. In Yuchau E. Hsiao \& Lian-hee Wee (eds.), Capturing phonological shades within and across 
languages. 142-165. Newcastle upon Tyne, Cambridge, UK: Cambridge Scholars Publishing.

Hsu, Huichuan. 1994. Constraint-based phonology and morphology: a survey of languages in China. San Diego, CA: University of California Dissertation.

Selkirk, Elisabeth. 1986. On derived domains in sentence phonology. Phonology Yearbook 3. 371-405.

Shih, Chilin. 1986. The prosodic domain of tone sandhi in Chinese. San Diego, CA: University of California Dissertation. 